\title{
Information System User Interface Design in Software Services Organization: A Small-Clan Case Study
}

\author{
Boonchoo Jitnupong*, and Waraporn Jirachiefpattana \\ Graduate School of Applied Statistics, National Institute of Development Administration, \\ Bangkok 10240, Thailand
}

\begin{abstract}
A user interface (UI) is one of the most significant parts of an information system (IS). A user-friendly UI helps users to carry out their tasks with efficiency, effectiveness, and satisfaction. UI consists of five elements: metaphor, mental model, navigation, interaction, and appearance. Based on these, the designer must carefully select and design each element to be appropriate for the users. This research examines the influential process named user-centered design (UCD), a well-known process to raise product quality. Furthermore, the roles UI elements in IS development by observing it in a small family software services company in Thailand. The findings of this study show small family team is proper with UI design that concern in usability for user. Even though some UCD processes or UI elements are quite unclear, the clan culture helps team to design UI with flexibility, and unbiassed obtain comments from user.
\end{abstract}

Key words: Clan organizational culture, user-centered design, user interface design, information system development

\section{Introduction}

The purpose of any product development is to create tools that enable users to carry out their jobs accurately and easily, and should be fun to interact with. Such is the definition of usability as is clearly laid out in the ISO9241-11 standard: "The extent to which a product can be used by specified users to achieves goals with effectiveness, efficiency, and satisfaction in a specific of use" [1]. In the same manner, a usable product helps users to be more productive by reducing human error and the learning curve. Moreover, usability helps to reduce user resistance by encouraging positive user, and client interaction.

In terms of an information system (IS), usability reflects the quality of its user interface (UI) [2], which is a significant component of an IS with the duty of being a translator between users, and the system. A UI provides the look (presentation) and feel (interaction) of a system [3], and a good UI will help users to carry out their tasks with satisfaction and productively [4]. To help develop an appropriate UI for user not for designer, User-centered design (UCD) has been recognized as a process that can help create an appropriate UI [5],

* Corresponding author: boonchoo.jit $@$ stu.nida.ac.th 
hence it is a development process that designers should follow. Moreover, when taking UI elements into consideration, it is important to mix the use of individual components to enable the designer to create a usable UI.

The characteristics of UCD, and UI elements design in small family software services firm are studied in this paper. To comprehend how a small-sized software services company designs appropriate UIs for its clients. The paper is ordered as follow. Next piece presents the knowledge background of the case study. The main concept are user interface design, and collaborative culture. The third section describes the case study methodology. In the following section is the results of findings. Last section is the conclusion that summarizes the result, and suggest the future research.

\section{User interface design}

To develop a usable UI, an IS development team should realize that users are a vital part of the team, and multi-disciplinary design team members are likely to raise useful ideas from many points of view. Clarifying UI requirements helps a team to understand what the system should be like in detail using design guidelines and standards that are trustworthy and easy to follow. UI and usability design can be separated into two styles: processoriented and product-oriented $[6,7]$, and software design tools assist development teams as well as UI evaluation by users and UI experts [8].

In this study, there are two parts in UI design: (i) the UI design process; (ii) the UI product characteristics.

\subsection{UI Design process}

From its beginning as an iterative and prototyping UI design approach, UCD has been developed to become a design method which ensures that the final product is developed from the users' standpoint and achieves the users' requirements; it is a method combining four main steps (see Figure 1).

First, understand and specify the context of use - this process is important towards understanding the range of physical, task social, organizational, and environmental aspects of the users. Systems analysts have a number of techniques to help understand the context of use, such as surveys, observation, task analysis, and interviewing. Second, specify the user and organizational requirements - designers and users must consider what the attributes of the UI requirements are, then the goals of each attribute, and finally the evaluation method for each attribute. The output of this stage is the UI requirements specifications - the combination of requirements and measuring the quality of the UI. Third, produce design solutions - the designers and users create a UI together that meets the requirements. Finally, evaluate the design against the requirements - this process will compare the quality of the UI with the original UI requirements to determine whether the latter have been met. If the results of the prototype evaluation meet the goals, the prototype can be used as the final product. On the other hand, if the prototype is not satisfactory, then the process must be started again. 


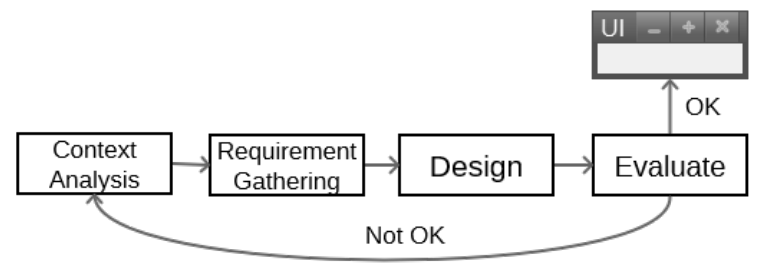

Fig. 1. The UCD process.

This study, an in-depth understanding of each step in this process was acquired from the case.

\section{$2.2 \mathrm{UI}$ design product characteristics}

In Marcus [9] provided the meaning of a user interface: "A computer-mediated means to facilitate communication between human beings or between a human being and an artifact. The user interface embodies both physical and communicative aspects of input and output, or interactive activity". Additionally, the UI consists of metaphors, mental models, navigation, interaction and appearance [9].

UI metaphors are computer-related elements that help users to understand, remember, and enjoy a UI. This means that the designer understands the users' needs and system objectives. Mental models are the scope of data, function, tasks, roles, and people. Navigation aids such as menus, windows, dialogue, or icons help movement through the mental model. Interactions include the techniques to receive input from the user and return the output to the user. Appearance or surface includes all perceptual attributes: visual, sound, colors, fonts, and animation (see Figure 2).

The objective of data gathering is to understand how a designer designs or selects each UI element on the basis of information from the users.

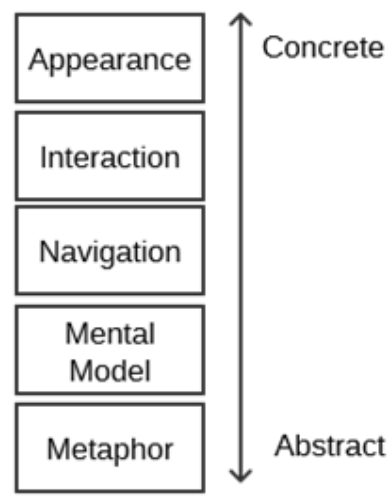

Fig. 2. Elements of UI 


\section{UI design in small clan company}

\subsection{Method}

The major data collection method in this research was by interview. During the interviews, the interviewer has two tasks: (i) to obtain answer from the previously prepared questions and (ii) ask the actual questions without bias [10]. At the same time, the data of the case is observed. Background sources of information such as an organizational chart, visions, mission, and samples of technical documents were also obtained from the respondents. Unfortunately, at this company, the work place was not static since employees also work from home or at client sites, and so data on their office working environment was not available. Moreover, by the nature of Startup Company, this company does not have a concrete visions or missions statement. So, these official evidences do not gather to analysis.

In order to understand the UI design in this company, the semi-structure questions were developed to interview the respondents. These questions are separated into two parts. The first part contained design process questions in order to understand their approach to UI design, and the second part was made up of questions on UI elements. If the interviewees were finding it difficult to understand parts of the process, the UI components examples were prepared to help identify their design preferences and to distinguish how they visualize UI elements.

The researcher interviewed with two team members. One man (Member-A), who is the team member and one woman (Member-B) who not only declared herself as a team member, but she is Project Manager also. In terms of experience, Member-B works in I.T. industries more than $10 \mathrm{yr}$. While, Member-A was work in another industry before (he worked in I.T. department). The researcher makes a contact them to make appointments and met them in February 2017.

\subsection{Case background}

The main mission of software services is to create tailor-made products for their clients, and, to carry this out well, software service consultants need to come up with useful ideas that help them create quality products for their clients. Service quality might affect company liquidity and enable it to operate in a market where competition is high. In the case of Thailand, in 2015, the Development Business Department, Ministry of Commerce, showed that software services industries (packages, consultants, and development) still comprised of more than 2500 firms [11]. Of these, almost all companies who are still operating are small- to medium-sized enterprises, and only a few have more than 200 employees.

The firm in this study has just recently been launched and thinks of itself as a Startup company. They do not have a physical workplace but instead use electronic communication tools such as collaborative software, instant messaging services, and/or email to brainstorm, work track, and talk within the team. IT consultancy is the key services of this team, and they organize exclusive software services development to serve clients' requirements. In terms of experience, their projects vary from ISs that assist governmental agencies, state enterprises, and private sector companies as well. Besides being expert in ISs, they are also skilled in web content systems. The predominant platform is web technology, both of fully open source and licensed. Even though today is the smartphone era, most ISs are still designed for the web platform. From the organizational culture theory, software development organization often be an adhocracy culture. The organizational culture which 
concern about innovative technology [12]. However, in this case, it is not follow these rules.

The design team at the company consisted of three members (two men and one woman) who are at least graduates with a Bachelor's degree in an IT field. Each of them is able to organize all of the IS tasks in the development process: analysis, design, coding, and testing. When designing, they apply their UI/UX (user experience) knowledge obtained from some of the classes when they studied, but most of their expertise comes from ten years or so of commercial experience. The team is small and very informal but its characteristics are diverse. Regardless of gender, personal feedback from these individuals concerning a UI design will probably result in a better design. Nevertheless, there is the risk that they might be similar in terms of knowledge because they graduated in the same or similar fields, but, more than anything, they are together as a family, like brothers and sisters.

A team characterized as "family" tends to share more than a job, and even without tradition, it is usually well consolidated. This behavior can contribute towards helping them share opinions openly. With regard to interactions between them and their customers, they will explicitly share knowledge together, which will aid in discovering techniques or tools that they think are good for the both the team and the users. The team are able to perform analyses in which the gained information can be presented to the users and applied to a project (this research must not effect to the main task). Therefore, they do not have a clear working framework. This characteristic alike with the group (family or clan) culture which the employees are concern about team relations and team spirits. They are less formalstructure control but love to decentralize the decision-making. They choose to push participation in team. So, everyone in the team are open-minded for others opinion. Moreover, the group culture often threat customer as an important part in team $[12,13]$.

"Personally, I think this situation is better (than my last organization). The formal framework should not become too habitual for an IT person." Member B said

Organizational size reflects upon the nature of the projects conceived by employees in the organization. Moreover, this might include the clients' network who are primarily concerned with the reliability of a service and/or product delivery. In contrast, working in a small team is more beneficial in terms of flexibility of service quality with more of an "as you like" attitude than big companies, which tend to be unwieldy.

Because users provide feedback about a UI at the same level as a core function, to design or rectify a UI is not easy and takes time. As a result, a design team should realize the importance of the UI design, and every member should agree that the UI is a vital part of the system that affects the success of the IS at the same level as a core system function.

\subsection{UI design process}

When applying UCD, the UI design process consists of four processes: users' context analysis, along with UI requirements development, prototyping, and evaluation. However, from the preliminary interviews, all four processes may not be clearly defined since they are blended into the UI design process by the design team, but not explicitly specified. An overview of the UI design process is shown in Figure 3. 


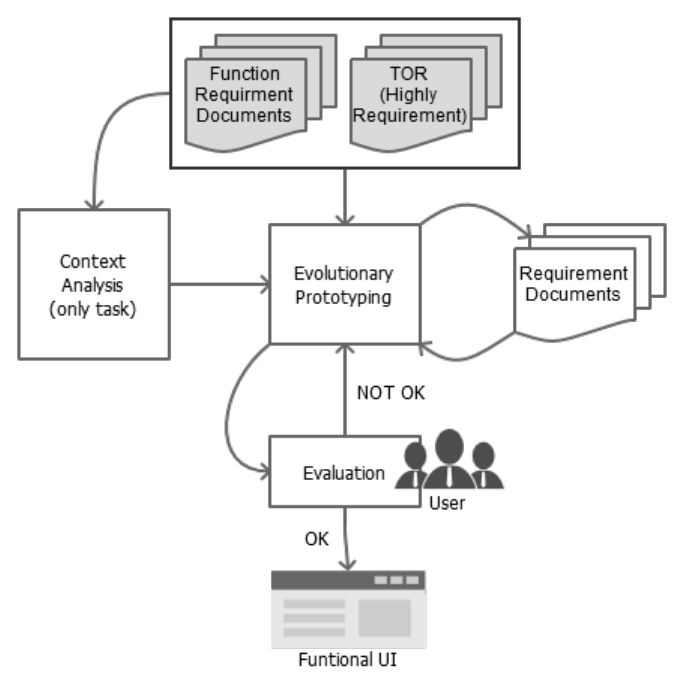

Fig. 3. UI design process.

\subsubsection{Understanding the users and their tasks}

During the process of context analysis, the team needs to analyze various aspects involving the users, tasks, and environment. In our case, the team surmised that they were to develop an IS for office use only, so they realized that the system should perform well in an office environment. However, they also took into account that the system should display correctly on any monitor size.

The characteristics of the users, such as gender and age, along with individual and cultural experience and knowledge affect user preferences, hence UI design. As a result, UI designers collect and analyze these factors in order to match the users' characteristics with the UI as closely as possible.

Unfortunately, as actually happened with this team, these data were not collected and analyzed clearly because user characteristics were estimated "by sight" during the requirements gathering process. Apparently, this oversight was not planned, but, in this case, the designers thought that the design was self-evident.

"We always do the task analysis." Member B said.

Task analysis is the process during which the team attempts to realize the business process or working steps involved in building the system, and the results of this process assist the designer to understand the data flow and structure of the system. Input-output of the system affects the navigation and interaction of the UI, and knowledge of this process can be translated into a diagrammatic view such as use case, data flow diagram, which describes the user interactions with the system and can be included in the system requirements.

From the above, both data on the user characteristics and task analysis can be used to create UI requirements. However, the team did not create a specific UI requirements document, but instead use the collected data to design a prototype which was then modified on the basis of the responses of the users in terms of primary need and aesthetics.

\subsubsection{Make it clear with a prototype}

"First impression of a user to the UI prototype will increase involvement, ownership, and satisfaction. As a result, the team can receive feedback on the pros/cons of the system easier 
and faster than just by talking or writing something down." They said in the same manner. This sentence tells the importance of a prototype to this team.

UI requirements development is a process that the team and users commit to with a UI prototype. If the users cannot see the system, it is not easy for them to give useful feedback, and so the prototype is a useful tool that helps in this endeavour. In this company, the UI requirements and prototype development are part of the same process. The prototype is an important part in identifying the system requirements. Creating a UI prototype as part of the requirements gathering process means that the team did not need to create a UI/usability requirements document, the creation of which they considered to be an inconvenience. Moreover, they preferred the development of the prototype as a means of identifying UI requirements so that they were not only generated from user supplied information. In addition, deliberation by the design team towards offering solutions might also help the users to accomplish their work more easily than at the present time. The team along with the clients committed to the UI theme together, where this theme provides the essentials of the prototype used in the design. Moreover, the users were able to interact with some parts of the system rather than trying to interpret its design from documents and/or drawings only.

"Prototyping takes a major role in every project since we have never built a system that fits with user requirements using analysis and design within a single iteration. The created prototype has usually become part of the real system," Member B said.

\subsubsection{Evaluation with the users}

The evaluation process during UI construction was not clearly methodologically determined for a person to perform. The team and the users enacted the UI evaluation together by the system testing process during unit testing, a test that the system meets with the requirements. During integration testing, the UI was evaluated by the design team from a technical perspective. Consequently, success during this process determined whether to commit to the UI design once more together with the users.

The participation of the users was important to the team, and the team requested that a user representatives participated in every UI prototype review. During each iteration, especially to provide feedback on the prototype, priority was given to users' feedback since they were the ones to determine whether a system is good or not.

One difficult problem that can occur is access to the actual users, which is something that is out of the team's control and might require a formal request to the owner of the project. It is important to have access to the real business users because trying to determine the nature of the user tasks should be a directed by their experience, and in order to understanding the importance of the system, they need to have enough knowledge about it.

As always, feedback is a shared process driven by the users' perspective. After the team obtain feedback, the development can be iterated, and so on. The completed UI needs to be equipped with the abilities of the final configuration, which is usually determined by a lot more variables than just the users, and so other people in the meeting room may be important too.

\subsection{UI design product characteristics}

In the case of UI characteristics, the design team predominantly work towards understanding the users' needs, designing a UI that helps them navigate the IS to carry out their business tasks, examining how the system "converses" with them, and determining the look and feel of the UI. Figure 4 shows the big picture of UI design product characteristics, 
and illustrates the design process from the bottom (abstract) to the top (concrete). In addition, Table 1 shows the method that this team used at each layer of the design process.

\subsubsection{Metaphors and user needs}

"A metaphor is a relation between language as an abstract system, individual language users, and cultural knowledge". When users encounter a user-interface metaphor, they bring their own context to the understanding and decoding of that metaphor. This context involves their culture, work, education, and all the qualities that identify them as an individual. Clearly, this is a major undertaking for the designer of a UI.

\subsubsection{Navigation design}

System navigation is guidance that helps user. A satisfactory system should guide the user from start to finish, which means that the team needed to understand each user task involved in the business processes in order to accomplish it. The information that describes the details of the users' business processes comes from work-flow analysis, and navigation of the processes is reflected in the structure of the system in terms of layout: template, position of buttons, system hierarchy, content organization, tabs, menu, and border in each UI.

A UI prototype contains the structure of the system, both the whole system shown on a sitemap or structure of the system and page structures or wireframes showing the structure of each page. Visualizing the system structure helps users to understand where they interact in the system - the places that they can go to and places that they already know. The wireframe is an useful tool if it makes sense, and can helps users to understand what they are currently doing and what are they should do next within the page.

Work-flow analysis helps design teams to separate main tasks from subtasks which the user must carry out to complete a main task. The design team designs a system so that the user carries out only one subtask at a time by ensuring that the UI guides the user to carry it out and either finish it or cancel it. This approach helps the users to be more productive than if trying to carry out more than one task at a time.

\subsubsection{Interaction design}

Interaction is communication through dialogue between the system and the users. Dialogue should be formal when used for input data validation messages so that error messages are straightforward and offer encouragement to solve the problem.

A good UI is to fully support users to complete their tasks in a short time with no crash," The interviewees said.

Additionally, interaction reflects the support level of the system and often depends on user expertise. Therefore, support level needs to be fully explained in the system requirements. In some systems, users need full support and need to perceive that the system politely informs them of any mistakes they make. On the other hand, users may require less support built in to the system because they think they are the skillful users. Usually, the design team constructs interactions along the middle path and tries to help the users by preventing, correcting, and recovering information when an error has occurred.

In term of language level, that reflects via wording in the system (dialog box, warning, validation message, system status bar etc.). The design team often use polite and formal word. In case of warning or validation message, dialog should show detail in passive way (the system have some mistakes). They think user will be more satisfactorily than aggressive system. 
Table 1. Summary of activities, method in UI design product characteristics.

\begin{tabular}{|c|c|c|c|c|}
\hline UI & Activities & Input & Method & Output \\
\hline 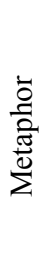 & $\begin{array}{l}\text { Understanding } \\
\text { Product Goal } \\
\text { (user needs) }\end{array}$ & $\begin{array}{l}\text {-Product } \\
\text { objective } \\
\text {-TOR } \\
\text {-Use Case }\end{array}$ & $\begin{array}{l}\text { - Group } \\
\text { Interviewing } \\
\text { - Document } \\
\text { Analysis } \\
\text { - Business } \\
\text { Process } \\
\text { Analysis }\end{array}$ & $\begin{array}{l}\text {-Strategic } \\
\text { Document } \\
\text { - Use Case } \\
\text { Diagram }\end{array}$ \\
\hline $\begin{array}{l}\frac{\bar{d}}{0} \\
\sum^{0} \\
\frac{\pi}{0} \\
\sum_{0}^{0}\end{array}$ & $\begin{array}{l}\text { Understanding } \\
\text { Users }\end{array}$ & $\begin{array}{l}\text {-User } \\
\text { Characteris- } \\
\text { ics } \\
\text {-User Tasks } \\
\text {-User } \\
\text { Environment }\end{array}$ & $\begin{array}{l}\text { - Group } \\
\text { Interview } \\
\text {-Task Analysis } \\
\text {-Document } \\
\text { Analysis } \\
\text {-Observation }\end{array}$ & $\begin{array}{l}\text {-Personas } \\
\text {-Use Case } \\
\text { Diagram } \\
\text { - Usage Scenario }\end{array}$ \\
\hline 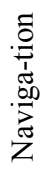 & $\begin{array}{l}\text { Information } \\
\text { Design }\end{array}$ & $\begin{array}{l}\text { Use Case } \\
\text { Diagram } \\
\text { Usage } \\
\text { Scenarios }\end{array}$ & $\begin{array}{l}\text { - Group } \\
\text { Interview } \\
\text { - Document } \\
\text { Analysis }\end{array}$ & $\begin{array}{l}\text {-System } \\
\text { Structure/ } \\
\text {-Site Map } \\
\text {-Wireframe }\end{array}$ \\
\hline \multirow{3}{*}{ 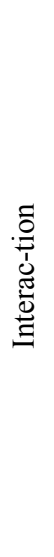 } & $\begin{array}{l}\text { User-system } \\
\text { Dialogue } \\
\text { Design }\end{array}$ & $\begin{array}{l}\text { Personas } \\
\text { Usage } \\
\text { Scenarios }\end{array}$ & $\begin{array}{l}\text { - User-System } \\
\text { Sequence } \\
\text { Analysis }\end{array}$ & $\begin{array}{l}\text {-System-user } \\
\text { Dialogue } \\
\text { Structure }\end{array}$ \\
\hline & $\begin{array}{l}\text { Error Handling } \\
\text { Design }\end{array}$ & $\begin{array}{l}\text {-Personas } \\
\text {-Function } \\
\text { Requirement } \\
\text {-Usage } \\
\text { Scenarios }\end{array}$ & $\begin{array}{l}\text {-Brainstorming } \\
\text {-Observation }\end{array}$ & $\begin{array}{l}\text {-Prevention } \\
\text { Message } \\
\text {-Correction } \\
\text { Message } \\
\text {-Recovery } \\
\text { Message }\end{array}$ \\
\hline & $\begin{array}{l}\text { Language } \\
\text { Usage }\end{array}$ & $\begin{array}{l}\text {-Personas } \\
\text {-Usage } \\
\text { Scenarios }\end{array}$ & $\begin{array}{l}\text {-Brainstorming } \\
\text {-Observation }\end{array}$ & $\begin{array}{l}\text {-Level of } \\
\text { Language } \\
\text { (Language } \\
\text { Theme) }\end{array}$ \\
\hline 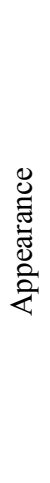 & $\begin{array}{l}\text { Apppearance } \\
\text { Design }\end{array}$ & $\begin{array}{l}\text {-Theme } \\
\text { (Color, } \\
\text { Fonts) } \\
\text {-Design } \\
\text { Guidelines } \\
\text {-Wireframe } \\
\text {-Prototype } \\
\text {-System-user } \\
\text { Dialogue } \\
\text { Structure } \\
\text {-Existing } \\
\text { System }\end{array}$ & $\begin{array}{l}\text {-Observation } \\
\text {-Brainstorming } \\
\text {-Exist System } \\
\text { Analysis } \\
\text {-Design and } \\
\text { Technology } \\
\text { Research }\end{array}$ & -UI theme \\
\hline
\end{tabular}




\subsubsection{Appearance and visual design}

An UI usually consists of many pages and it is important to keep a consistent style for each page. Therefore, the design team usually develop a template that every page will use. To this end, a wireframe is developed in the navigation design used. In addition, the design team usually choose consistent appearance elements such as background color, color palette, typography, and icon set appropriate for the users.

A designer's experience and knowledge appears on the prototype at the beginning of the design process because they bring their own experience to the table when creating a prototype. Comments from meetings between the team and the users is used to adjust it during the next design cycle.

"The team has experience of different types of users in different organizations, but the design during the first stage will be similar." Member B said

To select each appearance element, the design team first carries out research on the existing system and selects a theme that will become the basis for the new UI. Second, they use the successful structure to become the theme for headers, footers, left menu, and so on. Next, the color palette that they use is typically chosen from a theme from the user's organization. If the organization does not have one, the designers will probably use a bright color theme that is easy on the eye via a monitor.

Designers prefer to one icon set for menus and/or buttons because they think it increases a user's enjoyment when using the system as well as sets text and images to a good size for the current display. A point to be aware of during icon set selection is it must follow the chosen theme and be well conveyed. When selecting font size and typeface style, designers should be aware that most developed systems do not require formal fonts. However, text must be clear and legible. Official fonts are likely to be present in systems used by government agencies, as is usually clearly defined at the project start, but beyond that, needs to be clear and easy to read. Moreover, it is often the case that these attributes do not need to be changed at all.

Finally, the theme will be separate from other parts of the system, so if the design team need to change it, it only needs to be modified once for the entire system.

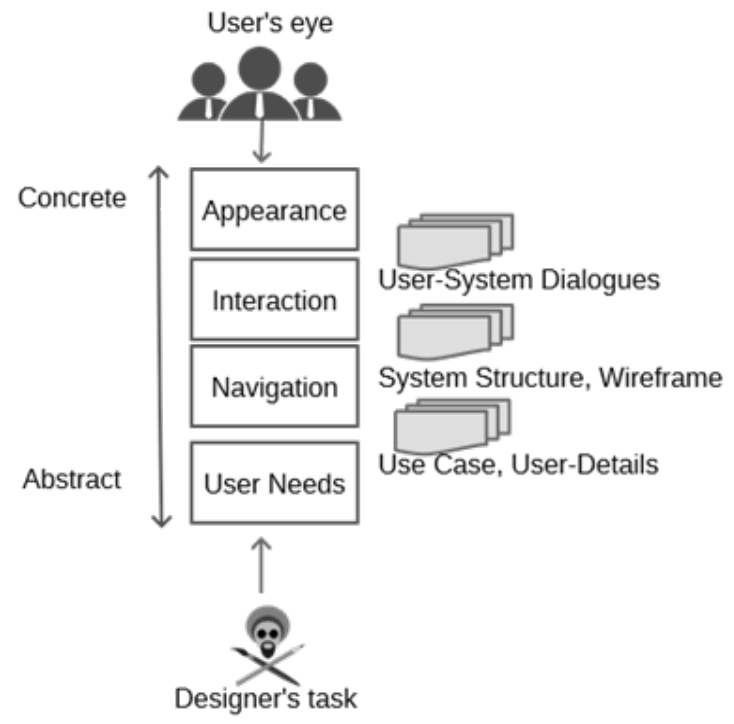

Fig. 4. UI design product characteristics. 


\section{Conclusion}

From the UCD approach and using UI elements, the design team carried out these tasks at a level that appropriate with the team for this specific project. Their main goals were to complete the project with quality and on-time. Initially, they may not have known what design the UIs should take and how to use UCD, but they understood that important contributions and positive responses from the users were crucial in this approach. Therefore, they paid special attention to the users as well as providing their prior knowledge and experience towards designing the system.

The characteristics of the organization and value supplied by the design team helped to optimize client satisfaction, and it is worth considering that survival of a business may be affected by the way the team designs the UIs. They perhaps tried to create the product too quickly by bypassing some of the traditional steps of systems analysis by using UCD. However, in their opinion, some parts of the UCD process, especially in environments with apparently clear UI requirements, can often be overlooked. In this case, it is possible that some important organizational information which might have influenced system usage, such as culture, behavior and business type were not included in the initial UI design analysis. These data may be very useful when they have to design a system for users in organizations where the UI design is not obvious. Nevertheless, small-clan teams are more flexible and suitable for UI designs that incorporate user values than big teams. They share techniques and are open-minded when receiving feedback within the team and especially from the users.

The key lesson from this study is partial UCD and some UI elements, already recognized by software services teams, were an excellent approach in this case since the design team understood that the UI is a vital part of a successful IS. In addition, the design team understood that activities, input, output and how to query data affected the design of usable UIs. However, some user information was not properly gathered prior to the analysis and design phase and so the design team might have been better off employing UI design guidelines to help them clarify the activities and characteristics of the UI design. Consequently, they seem to be need the combinations of methods which not only UCD with abstract UI elements but also another accepted IS development process.

The researcher would like to thank Khun Kanyanut Homsapaya and Siriwatthapurich Co.,Ltd. with their useful information and comments that were used in this article.

\section{References}

1. ISO 9241-210. Ergonomics of human-system interaction - Part 210: Human-centered design for interactive systems [Online] from https://www.iso.org/standard/52075.html (2010). [Accessed on 12 August 2017]

2. J.R. Lewis. Int J Hum Comput Interact., 30,9:663-684 (2012). http://www.tandfonline.com/doi/abs/10.1080/10447318.2014.930311

3. E. Callahan. Annu. Rev. Inf. Sci. Technol., 39,1:255-310 (2005). https://kinasevych.ca/2010/04/28/callahan-2005-interface-design-and-culture/

4. J. Nielsen. Usability 101: introduction to usability [Online] from https://www.nngroup.com/articles/usability-101-introduction-to-usability/ (2012). [Accessed on 12 August 2017]

5. H. Kikuchi, S. Kimura, S. Ohkubo, H. Inamura, A. Takeshia. NTT DOCOMO Tech. J., 12,3:33-41 (2010). https://www.nttdocomo.co.jp/english/binary/pdf/corporate/technology/rd/technical jour nal/bn/vol12_3/vol12_3 033en.pdf 
6. E. Folmer, J. Bosch. J. Syst. Softw., 70,1-2:61-78 (2004). https://dl.acm.org/citation.cfm?id=2305799

7. T. Keinonen. One-dimensional usability influence of usability on consumers' product preference. Helsinki: University of Art and Design (1998). p. 311 https://books.google.co.id/books/about/One_dimensional_Usability.html?id=q7ZuAAA ACAAJ\&redir esc $=y$

8. B. Shneiderman, C. Plaisant. Designing the user interface: strategies for effective human-computer interaction. 5th edition. USA: Addison-Wesley (2010). p.121 https://www.amazon.com/Designing-User-Interface-Human-ComputerInteraction $/ \mathrm{dp} / 0321537351$

9. A. Marcus. Interactions, 9,5:19-24 (2002). https://dl.acm.org/citation.cfm?id=566992

10. R.K. Yin. Case study research: design and method. USA: SAGE Publications (2013). P.110 https://www.amazon.com/Case-Study-Research-MethodsApplied/dp/1452242569

11. Department of Business Development. Business Data Warehouse [Online] from http://datawarehouse.dbd.go.th/bdw/business/index.html (2016). [Accessed on 5 December 2016]

12. K.S. Cameron, R.E. Quinn. Diagnosing and changing organizational culture: based on the competing values framework. USA: Jossey-Bass (2011). p. 49 https://www.amazon.com/Diagnosing-Changing-Organizational-CultureCompeting/dp/0470650265

13. J. Suderman. J Pract Consult., 4,1:52-58 (2012). https://www.regent.edu/acad/global/publications/jpc/vol4iss1/JPC_Vol4Iss1.pdf\#page= $\underline{59}$ 